\title{
Systematic design of 3D auxetic lattice materials with programmable Poisson's ratio for finite strains
}

\section{Wang, Fengwen}

Published in:

Journal of the Mechanics and Physics of Solids

Link to article, DOI:

10.1016/j.jmps.2018.01.013

Publication date:

2018

Document Version

Peer reviewed version

Link back to DTU Orbit

Citation $(A P A)$ :

Wang, F. (2018). Systematic design of 3D auxetic lattice materials with programmable Poisson's ratio for finite strains. Journal of the Mechanics and Physics of Solids, 114, 303-318.

https://doi.org/10.1016/j.jmps.2018.01.013

\section{General rights}

Copyright and moral rights for the publications made accessible in the public portal are retained by the authors and/or other copyright owners and it is a condition of accessing publications that users recognise and abide by the legal requirements associated with these rights.

- Users may download and print one copy of any publication from the public portal for the purpose of private study or research.

- You may not further distribute the material or use it for any profit-making activity or commercial gain

- You may freely distribute the URL identifying the publication in the public portal

If you believe that this document breaches copyright please contact us providing details, and we will remove access to the work immediately and investigate your claim 


\title{
Systematic design of 3D auxetic lattice materials with programmable Poisson's ratio for finite strains
}

\author{
Fengwen Wang* \\ Department of Mechanical Engineering, Technical University of Denmark \\ Nils Koppels Allé, Building 404, 2800 Kgs. Lyngby, Denmark
}

\begin{abstract}
This paper presents a systematic approach for designing 3D auxetic lattice materials, which exhibit constant negative Poisson's ratios over large strain intervals. A unit cell model mimicking tensile tests is established and based on the proposed model, the secant Poisson's ratio is defined as the negative ratio between the lateral and the longitudinal engineering strains. The optimization problem for designing a material unit cell with a target Poisson's ratio is formulated to minimize the average lateral engineering stresses under the prescribed deformations. Numerical results demonstrate that 3D auxetic lattice materials with constant Poisson's ratios can be achieved by the proposed optimization formulation and that two sets of material architectures are obtained by imposing different symmetry on the unit cell. Moreover, inspired by the topology-optimized material architecture, a subsequent shape optimization is proposed by parametrizing material architectures using super-ellipsoids. By designing two geometrical parameters, simple optimized material microstructures with different target Poisson's ratios are obtained. By interpolating these two parameters as polynomial functions of Poisson's ratios, material architectures for any Poisson's ratio in the interval of $v \in[-0.78,0.00]$ are explicitly presented. Numerical evaluations show that interpolated auxetic lattice materials exhibit constant Poisson's ratios in the target strain interval of $[0.00,0.20]$ and that $3 \mathrm{D}$ auxetic lattice material architectures with programmable Poisson's ratio are achievable.
\end{abstract}

Keywords: 3D auxetic lattice material, finite strain, programmable Poisson's ratio, shape optimization, topology optimization

\section{Introduction}

Novel materials with special properties are highly attractive for modern technology, such as materials with exotic mechanical properties [1, 2, 3], materials with enhanced electric, magnetic properties [4, 5] and acoustic meta materials exhibiting negative refraction [6, 7]. One such exotic material property is the negative Poisson's ratio [1]. In

${ }^{*}$ Corresponding author. Tel. :+45 4525 4266; Fax: +45 45931475.

Email address: fwan@mek.dtu.dk (Fengwen Wang) 
contrast to conventional materials with positive Poisson's ratio, materials with negative Poisson's ratio (NPR), known as auxetic materials, will expand laterally instead of shrinking when stretched. Auxetic materials have been claimed to exhibit enhanced mechanical properties, such as shear or indentation resistance [8, 9], fracture toughness [10], extraordinary damping properties [11,12,13], etc. Hence auxetic materials exhibit potential applications in many fields [14, 15].

Besides natural auxetic materials, found in some rocks and minerals, a wide variety of auxetic materials have been created and fabricated using different deformation mechanisms [16], including polymeric and metallic foams [1], microporous polymers [2, 17], carbon fiber laminates [18], honeycomb structures [19, 20], microelectromechanical systems [21], etc. Moreover, topology optimization methods [22] have been successfully employed to create auxetic materials [23, 24], by optimizing the material layouts in unit cells. Optimized auxetic materials have been experimentally verified [21, 25]. Furthermore, different techniques have been developed in topology optimization methods to achieve manufacturability and control of minimum feature sizes of the topology optimized designs [26, 27, 28, 29, 30, 31].

Most of the research in auxetic materials has so far been mainly focused on 2D linear material for small deformations. However, experiments have demonstrated that auxetic materials are highly sensitive to deformation and exhibit nonlinear Poisson's ratio profile under finite strains [4, 9, 19, 32]. A challenge in topology optimization methods is numerical instabilities when handling finite strains. The numerical instabilities are induced by excessive deformations in the low stiffness regions appearing in the topology optimization process [33]. Recently, an energy interpolation scheme has been proposed to circumvent numerical instabilities for finite strain problems [34], and bared here on, 2D auxetic materials have been designed for finite strains using topology optimization [35]. Inspired by the optimized configurations in [35], systematic parametrizations of materials with various Poisson's ratio values for finite strains have been realized and experimentally verified. Moreover, 2D materials with any given Poisson's ratio in an interval of $[-0.8,0.8]$ can be obtained directly by geometrical parameter interpolations using just a few control points [36].

Previously work on auxetic materials has been focused on 2D scenarios, however recent advances in manufacturing technologies, such as 3D printing, make fabrication of 3D artificial complex auxetic material attainable. 3D auxetic materials have been fabricated using metal, polyamide and rubber [25, 37, 38, 39, 40]. Babaee et al. [37] proposed 3D buckling-induced auxetic materials, i.e. bucklicrystal. The proposed bucklicrystals have been fabricated with a soft silicone-based rubber using additive manufacturing. It has been numerically and experimentally verified that bucklicrystals are capable of retaining auxetic properties at finite strains. Ren et al. [38] further studied the buckling-induced auxetic material patterns by fabricating them using metallic materials. Recently, Huang, et al. [39] studied 3D antitetrachiral auxetic materials both numerically and experimentally by fabricating them in rubber-like material using 3D additive manufacturing. Moreover, Andreassen, et al . [25] designed 3D isotropic auxetic materi- 
als for small deformations based on homogenization methods and experimentally verified the material performance. Cabras and Brun [40] proposed 3D auxetic lattices for small deformations consisting of slender cross-shape and truss elements, where auxetic behavior was induced by the stiffness contrast of component elements and linear auxetic lattices were obtained with tunable Poisson's ratio as a function of the stiffness contract ratio. However the systematic design approach for 3D auxetic materials for finite strains is still unaccounted for due to high computational cost and complexities.

This study extends the work in 2D auxetic material design [35] to 3D auxetic material design utilizing a flexible framework for large scale topology optimization based on the Portable, Extensible Toolkit for Scientific Computation (PETSc) [41, 42]. Under finite strains, the effective material properties are dependent on the deformations and have to be evaluated for each deformation state. Hence, the calculation of the effective material properties under finite strains results in computationally intensive nonlinear homogenization [43]. To circumvent the cumbersome homogenization of materials under finite deformations, this study focuses on material design under numerical tensile tests, where the material is uniformly stretched along each of the three principal directions sequentially and the Poisson's ratios calculated for each case. A 3D unit cell model is proposed to mimic the compression and tensile tests. Based on the proposed model, the Poisson's ratios of the material are defined as the negative ratio between the engineering strains in the direction perpendicular to the loading direction and the engineering strain along the loading direction. In the proposed model, the averaged lateral engineering stresses vanish when the unit cell deformations correspond to the material Poisson's ratios. Hence it is possible to formulate the design problem as an optimization problem with the goal of minimizing the lateral engineering stresses under a series of given deformations obtained using the target Poisson's ratio in a design strain interval. The geometrically nonlinear behavior of the periodic unit cell is simulated using a total Lagrangian finite element formulation [44], and solved by a Newton-Raphson procedure. 3D auxetic materials with different symmetries are designed using the proposed approach. Moreover, inspired by the topology optimized material configurations, a subsequent shape optimization approach is proposed, where material architectures are parametrized using super-ellipsoids tuned by several control points. Based on the shape-optimized materials, material architectures for any Poisson's ratios between -0.78 and 0.0 can be obtained by interpolating two geometrical parameters, and corresponding performances are evaluated.

The paper is organized as follows. Section 2 presents the unit cell model which mimics the compression and tensile test, and formulates the optimization problem for designing materials with a prescribed Poisson's ratio under finite strains. Section 3 first presents topology optimized materials for different target Poisson's ratios and corresponding performance. Then, inspired by the optimized material configurations, a shape optimization scheme is proposed. Finally, material architectures for any Poisson's ratio between -0.78 and 0.0 are explicitly presented by interpolating 
the shape optimized material configurations. Section 4 is dedicated to discuss fabrication of materials using 3D printing. The paper ends with conclusions in Section 5.

\section{Optimization formulation of $3 D$ auxetic material design}

This section presents the optimization formulation for designing materials with constant Poisson's ratios under finite strains using topology optimization. To simplify the problem, this paper only considers material design under compression and tensile tests.

\subsection{Characterization of material properties}

The section is focused on establishing a unit cell model to characterize material properties in tensile or compression tests under finite strains, including Poisson's ratio and material stiffness.

In the compression or tensile test, the specimen is uniformly compressed or stretched under a uniaxial load along one principal direction (here the $x$-direction) as shown in the left panel in Fig. 1. By measuring the lateral engineering strains $\left(\varepsilon_{22}^{1}, \varepsilon_{33}^{1}\right)$ and the longitudinal engineering strain $\varepsilon_{11}^{1}$, the material stiffness and Poisson's ratios are defined by

$$
E_{11}=\frac{\sigma_{11}^{1}}{\varepsilon_{11}^{1}}, \quad v_{12}=-\frac{\varepsilon_{22}^{1}}{\varepsilon_{11}^{1}}, \quad v_{13}=-\frac{\varepsilon_{33}^{1}}{\varepsilon_{11}^{1}}
$$

where the superscript indicates the compression or tensile direction with $\square^{1}, \square^{2}, \square^{3}$ representing the $x$-, $y$ - and $z$ direction, respectively, $\sigma_{11}^{1}$ is the longitudinal engineering stress in the $x$-direction and it is defined by $\sigma_{11}^{1}=\frac{P}{A}$ with $P$ as the longitudinal load and $A$ as the initial area of the face perpendicular to the $x$-direction. $\varepsilon_{11}^{1}$ is the longitudinal engineering strain, and $\varepsilon_{22}^{1}$ and $\varepsilon_{33}^{1}$ are the lateral engineering strains in the $y$ - and $z$-direction, respectively. It is worthwhile mentioning that the lateral engineering stresses, $\sigma_{22}^{1}$ and $\sigma_{33}^{1}$ on faces perpendicular to the $y$-and $z$-axis, respectively, are zero as illustrated in the left panel in Fig. 1

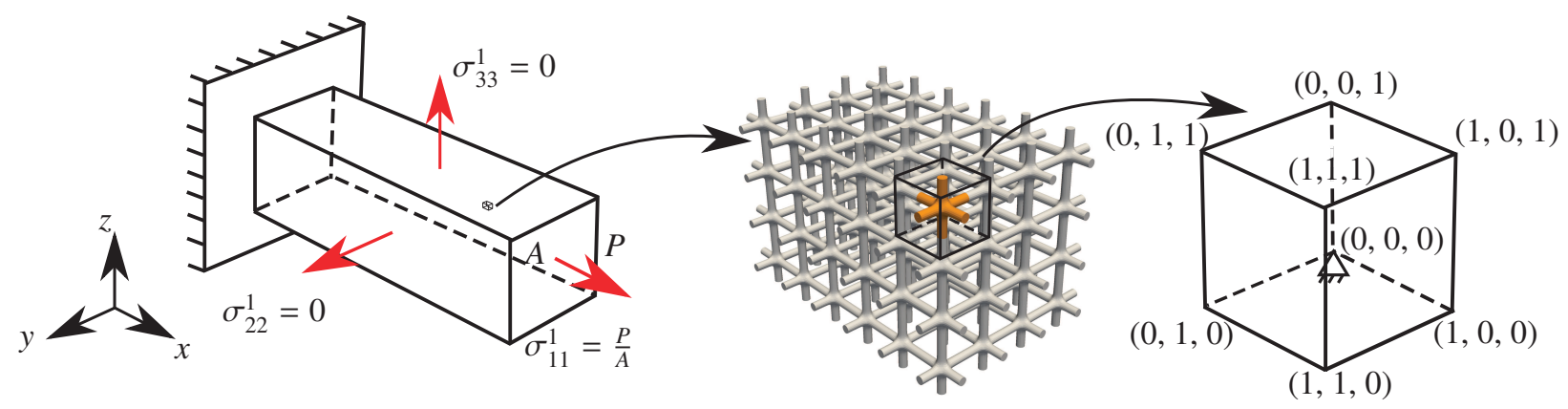

Figure 1: Schematic illustration of compression or tensile tests, illustration of the representative volume element (RVE, i.e. unit cell) and corresponding boundary conditions to mimic compression and tensile tests. 
The material properties can be characterized using a representative volume element (RVE, i.e., unit cell). The RVE is defined as the smallest volume where the material properties can be measured, see the middle panel in Fig. 1 . This paper focuses on periodic materials, hence the RVE is a periodic microstructure in the material and the cell size is normalized to be unit, as shown in the right panel. To mimic compression or tensile tests using the representative unit cell, special boundary conditions are imposed on the unit cell. The displacement vectors $(u, v, w)$ on the three pairs of faces, i.e. the face pairs perpendicular to the $x$-, $y$ - and $z$-axis, are defined by,

$$
\begin{array}{lll}
\left.(u, v, w)\right|_{x=0}=\left(u_{1}, v_{1}, w_{1}\right), & \left.(u, v, w)\right|_{x=1}=\left(u_{2}, v_{2}, w_{2}\right) \\
\left.(u, v, w)\right|_{y=0}=\left(u_{3}, v_{3}, w_{3}\right), & \left.(u, v, w)\right|_{y=1}=\left(u_{4}, v_{4}, w_{4}\right) \\
\left.(u, v, w)\right|_{z=0}=\left(u_{5}, v_{5}, w_{5}\right), & \left.(u, v, w)\right|_{z=1}=\left(u_{6}, v_{6}, w_{6}\right)
\end{array}
$$

where $u, v$ and $w$ are the displacements along the $x-, y$-, and $z$-axis, respectively. The unit cell is fully constrained at one point, and the boundary conditions imposed on the three pairs of faces are stated in Eq. (3),

$$
\begin{array}{lll}
u_{2}-u_{1}=\Delta u, & v_{2}-v_{1}=0, & w_{2}-w_{1}=0 \\
u_{4}-u_{3}=0, & v_{4}-v_{3}=\Delta v, & w_{4}-w_{3}=0 \\
u_{6}-u_{5}=0, & v_{6}-v_{5}=0, & w_{6}-w_{5}=\Delta w
\end{array}
$$

In the unit cell model, a compression or tensile test along the $x$-axis illustrated in the left panel in Fig. 1 can be also mimicked by prescribing the longitudinal engineering strain, $\varepsilon_{11}^{1}=\Delta u$. From Eq. (1), the Poisson's ratio $v_{12}$ is defined as negative ratio between the lateral strain in the $y$-direction and the prescribed longitudinal strain, i.e. $v_{12}=-\frac{\Delta v}{\Delta u}$. Similarly the Poisson's ratio $v_{13}$ is defined as $v_{13}=-\frac{\Delta w}{\Delta u}$. Following the same approach with the longitudinal strain direction being along $y$ - or $z$ - axis, the Poisson's ratios of the material in all directions are calculated by,

$$
\begin{array}{llll}
v_{12}=-\frac{\Delta v}{\Delta u}, & v_{13}=-\frac{\Delta w}{\Delta u}, & \varepsilon_{11}^{1}=\Delta u, & \text { with prescribed } \Delta u \\
v_{21}=-\frac{\Delta u}{\Delta v}, & v_{23}=-\frac{\Delta w}{\Delta v}, & \varepsilon_{22}^{2}=\Delta v, & \text { with prescribed } \Delta v \\
v_{31}=-\frac{\Delta u}{\Delta w}, & v_{32}=-\frac{\Delta v}{\Delta w}, & \varepsilon_{33}^{3}=\Delta w, & \text { with prescribed } \Delta w
\end{array}
$$

It is stressed that this study employs the secant Poisson's ratio shown in Eqs. (4) - 6), as opposed to the tangential formulation in which the Poisson's ratios are defined as $v_{12}=-\frac{\partial \varepsilon_{22}^{1}}{\partial \varepsilon_{11}^{1}}$. Since this paper aims at providing deformationindependent auxetic materials, i.e., constant Poisson's ratio, the two formulations are identical for the optimized 
materials.

The material stiffness is characterized using averaged lateral engineering stress, defined by,

$$
\bar{\sigma}_{11}^{i}=\frac{\int_{s \mid x=1} \sigma_{11}^{i} d s}{\int_{s \mid x=1} d s}, \quad \bar{\sigma}_{22}^{i}=\frac{\int_{s \mid y=1} \sigma_{22}^{i} d s}{\int_{s \mid y=1} d s}, \quad \bar{\sigma}_{33}^{i}=\frac{\int_{s \mid z=1} \sigma_{33}^{i} d s}{\int_{s \mid z=1} d s}, i \in\{1,2,3\}
$$

where $s$ represents an area in the unit cell and is defined as $s \equiv\{(x, y, z) \mid(x, y, z) \in s\}$, and $i$ indicates the longitudinal direction of the compression or tensile tests.

The material stiffness is defined by,

$$
E_{11}=\frac{\bar{\sigma}_{11}^{1}}{\varepsilon_{11}^{1}} \text { with prescribed } \Delta u ; \quad E_{22}=\frac{\bar{\sigma}_{22}^{2}}{\varepsilon_{22}^{2}}, \text { with prescribed } \Delta v ; \quad E_{33}=\frac{\bar{\sigma}_{33}^{3}}{\varepsilon_{33}^{3}}, \text { with prescribed } \Delta w
$$

Based on the proposed unit cell model, one can set the strains of the unit cell in all three principal directions. If the lateral deformations are equal to $\Delta v=-v_{12} \Delta u$ and $\Delta w=-v_{13} \Delta u$, the averaged lateral engineering stresses, $\bar{\sigma}_{22}^{1}$ and $\bar{\sigma}_{33}^{1}$, vanish. In this approach, the Poisson's ratio of the material can be obtained by iteratively updating the unit cell deformations such that the averaged lateral engineering stresses vanish. When the averaged lateral engineering stresses are equal to zero, the Poisson's ratios of materials are obtained using Eq. (4).

Alternatively, one can prescribe the longitudinal strain along the $x$-axis as $\varepsilon_{11}^{1}$, and calculate the lateral engineering strains represented by $-\Delta v$ and $-\Delta w$ by solving the static equilibrium of the unit cell, as in the $2 \mathrm{D}$ case in [35]. The corresponding Poisson's ratios can be calculated using Eq. (4).

The model problem is discretized using the finite element method [44], and the static equilibrium of the unit cell under finite deformations is governed by the following equation,

$$
\boldsymbol{r}=\boldsymbol{f}^{\mathrm{ext}}-\boldsymbol{f}^{\mathrm{int}}(\boldsymbol{u})=0
$$

where $\boldsymbol{r}$ is the residual nodal force vector, $\boldsymbol{f}^{\text {ext }}$ is the external nodal force vector resulting from the prescribed deformation, $\boldsymbol{f}^{\text {int }}(\boldsymbol{u})$ is the internal nodal force vector that is dependent on the nodal displacement vector, $\boldsymbol{u}$. The internal nodal force vector is defined by,

$$
\boldsymbol{f}^{\mathrm{int}}(\boldsymbol{u})=\frac{\partial\left(\int_{v} W(\boldsymbol{u}) d v\right)}{\partial \boldsymbol{u}}
$$

where $W(\boldsymbol{u})$ is the stored elastic energy density describing material behavior. The detailed calculation of $\boldsymbol{f}^{\text {int }}(\boldsymbol{u})$ can be found e.g. in [44].

The static equilibrium, Eq. (99), is solved iteratively using the Newton-Raphson method, with the incremental 
equation given by,

$$
\boldsymbol{K}_{t} \Delta \boldsymbol{u}=\boldsymbol{r}
$$

where the nodal displacement vector is updated by $\boldsymbol{u}=\boldsymbol{u}+\alpha \Delta \boldsymbol{u}$ with $\alpha=0.5$ to stabilize the Newton-Raphson iterations, and $\boldsymbol{K}_{t}=-\partial \boldsymbol{r} / \partial \boldsymbol{u}$ is the tangent stiffness matrix. The formulation of the tangent stiffness matrix is described in standard books on non-linear finite element theory, e.g. [44].

The average engineering stresses in Eq. (7) are calculated using the internal forces by

$$
\bar{\sigma}_{11}^{i}=\frac{\int_{s \mid x=1} \sigma_{11}^{i} d s}{\int_{s \mid x=1} d s}=\frac{\sum_{s \mid x=1} f_{i}^{\mathrm{int}}\{1\}}{\int_{s \mid x=1} d s}, \quad \bar{\sigma}_{22}^{i}=\frac{\int_{s \mid y=1} \sigma_{22}^{i} d s}{\int_{s \mid y=1} d s}=\frac{\sum_{s \mid y=1} f_{i}^{\mathrm{int}}\{2\}}{\int_{s \mid y=1} d s}, \quad \bar{\sigma}_{33}^{i}=\frac{\int_{s \mid z=1} \sigma_{33}^{i} d s}{\int_{s \mid z=1} d s}=\frac{\int_{s \mid z=1} f_{i}^{\mathrm{int}}\{3\}}{\int_{s \mid z=1} d s}
$$

where indices in the brackets, $\{\square\}$, indicate the component of the internal force along the indicated principle direction.

\subsection{Design parametrization}

The configuration of a unit cell is described by the material distribution in the unit cell. In topology optimization methods, an element-wise constant design variable $\rho_{e}$ is assigned to element $e$. A physical density $\bar{\rho}_{e}$ is introduced as a function of $\rho$ to represent the material occupation in element $e$. The element, $e$, is occupied by base material when $\bar{\rho}_{e}=1$ and by void when $\bar{\rho}_{e}=0$. The elemental property is interpolated using the solid isotropic material with penalization (SIMP) model [45], stated by,

$$
E_{e}=\bar{\rho}_{e}^{p}\left(E_{1}-E_{0}\right)+E_{0}
$$

where $E_{1}$ is the Young's modulus of the base material, $E_{0}=10^{-9} E_{1}$ is employed to represent void regions to avoid numerical singularities in the finite element analysis, and $p=3$ is chosen as the standard penalization factor. In order to enhance the discreteness of the optimized materials, a hyperbolic tangent threshold projection is employed to generate the physical density, $\bar{\rho}_{e}$ [31], calculated by,

$$
\bar{\rho}_{e}=\frac{\tanh (\beta \eta)+\tanh \left(\beta\left(\widetilde{\rho}_{e}-\eta\right)\right)}{\tanh (\beta \eta)+\tanh (\beta(1-\eta))}
$$

with $\widetilde{\rho}_{e}$ as the filtered design variable calculated using a PDE filter presented in [46]. The PDE filter is employed to prevent checkerboard patterns and mesh-dependency of optimized designs in topology optimizations. When $\beta$ is big, $\bar{\rho}_{e} \approx 1$ if $\widetilde{\rho}_{e}>\eta$ and $\bar{\rho}_{e} \approx 0$ if $\widetilde{\rho}_{e}<\eta$. Hence the projection in Eq. (14) suppresses the gray element density regions induced by the PDE filter when $\beta$ is big and ensures black-white designs in the optimization process. Moreover, it mimics the manufacturing process, and can be used in the robust design formulations [31], where the manufacturing 
errors can be taken into account by choosing different thresholds, $\eta$.

In order to ensure black-white designs, a continuation scheme is employed to increase $\beta$ in Eq. 114. In this paper, $\beta$ is updated every 40 iterations using $\beta \leftarrow 2 \beta$, with a maximal value of $\beta_{\max }=50$ and an initial value of $\beta=4$.

To avoid the numerical instabilities induced by low stiffness regions, i.e. low physical density elements, an energy interpolation formulation presented in [34] is employed. Therein, low stiffness regions are modelled using linear elastic elements. In this paper, all the regions with $E_{e}<0.1 E_{1}$ are treated as low stiffness regions. The reader is referred to [34] for further details.

As was done in [35], we use a hyperelastic material model to describe the material properties of the base material. The material behavior is described by a modified St. Venant-Kirchhoff model following the study from [47], given as:

$$
W=\frac{1}{2} \lambda(J-1)^{2}+\frac{\mu}{4}\left(I I_{C}-2 I_{C}+3\right)
$$

Here $\lambda$ and $\mu$ are the Lamé parameters of materials, $J=\operatorname{det}(\boldsymbol{F})$ with $\boldsymbol{F}$ as the deformation gradient, $I_{C}=\operatorname{tr}(\boldsymbol{C})$ with $\boldsymbol{C}=\boldsymbol{F}^{T} \boldsymbol{F}$ as right Cauchy strain, and $I I_{C}=\operatorname{tr}\left(\boldsymbol{C}^{2}\right)$.

\subsection{Design problem formulation}

This paper aims at designing materials with constant Poisson's ratios in a tensile test under finite deformations. The design problem can be formulated directly as the minimization of the error between actual and prescribed Poisson's ratios for a given strain range as in [35], where one prescribes the longitudinal stretching strain and extracts the actual Poisson's ratio based on the calculated lateral engineering strains (see Eqs.(47-(6)). From a high-performance computing standpoint however, this approach causes undesirable computational complexity due to the need for communication between nodes. Instead of solving for Poisson's ratios, one can prescribe the unit cell deformation, i.e., the engineering strain vector $[\Delta u, \Delta v, \Delta w]=\left[\varepsilon,-v^{*} \varepsilon,-v^{*} \varepsilon\right]$ with $\varepsilon \in[0, \bar{\varepsilon}]$ to mimic tensile tests along the $x$-axis. If the actual Poisson's ratio of the material equals the target one, $v^{*}$, the average lateral engineering stresses on the faces parallel to the uniaxial loading direction, i.e. $\bar{\sigma}_{22}^{1}$ and $\bar{\sigma}_{33}^{1}$, vanish as mentioned in Section 2.1. Likewise, the scheme also works for the tensile tests along the $y$-and $z$-axis. Hence, the optimization objective function for designing materials with a constant Poisson's ratio under finite deformations is formulated to minimize the maximum averaged lateral engineering stresses while employing a series of prescribed engineering strain vectors generated using the target Poisson's ratio $\left(v^{*}\right)$ for a given longitudinal strain interval of $\varepsilon \in[0, \bar{\varepsilon}]$. The min-max formulation ensures that Poisson's ratio variation of the optimized materials is highly restrained. In order to ensure the material stiffness, lower 
bound constraints are employed to the longitudinal stress. The optimization formulation is given as

$$
\begin{aligned}
\min _{\boldsymbol{\rho}} \max _{\boldsymbol{\varepsilon}^{i m}} \quad & f=\left(\frac{\bar{\sigma}_{j j}^{i}\left(\boldsymbol{\varepsilon}^{i m}\right)}{\varepsilon_{i i}^{i m}}\right)^{2} \quad\{j: j \in\{1,2,3\} \text { and } \mathrm{j} \neq \mathrm{i}\}, i \in\{1,2,3\}, 1 \leq m \leq M \\
\text { s.t. } \quad & \boldsymbol{r}=0 \\
& \bar{\sigma}_{i i}^{i}\left(\boldsymbol{\varepsilon}^{i m}\right)>\sigma^{*} \\
& \boldsymbol{v}^{T} \overline{\boldsymbol{\rho}}(\boldsymbol{\rho}) \leq g^{*} \\
& \mathbf{0} \leq \boldsymbol{\rho} \leq \mathbf{1}
\end{aligned}
$$

where the index, $i$, denotes the uniaxial loading direction in a tensile test, $\boldsymbol{\varepsilon}^{i m}$ is the $m$ th prescribed engineering strain vector in the target longitudinal strain interval considered, the stress, $\bar{\sigma}_{j j}^{i}\left(\varepsilon^{i m}\right)$, is normalized with respect to the longitudinal strain, $\varepsilon_{i i}^{i m}, M$ is the total number of the longitudinal strain points considered in the design strain interval, the target Poisson's ratio, $v^{*}$, is implicitly enclosed by the prescribed strain vectors as described in the previous section, $\sigma^{*}$ is the prescribed lower bound on the longitudinal stress along the tensile test direction to ensure material stiffness and $g^{*}$ is the upper bound of volume constraint.

Based on design parametrization of the unit cell, the adjoint method is employed to calculate the sensitivities of the objective and constraint functions, $\phi$, with respect to a design variable $\rho_{e}$, given as:

$$
\frac{\partial \phi}{\partial \rho_{e}}=\frac{\partial \phi(\boldsymbol{u}, \boldsymbol{\rho})}{\partial \rho_{e}}+\lambda^{T} \frac{\partial \boldsymbol{r}}{\partial \rho_{e}}
$$

with $\lambda$ being the adjoint variable vector, which is obtained by solving

$$
\left(\boldsymbol{K}_{t}\right)^{T} \lambda=-\left(\frac{\partial \phi(\boldsymbol{u}, \boldsymbol{\rho})}{\partial \boldsymbol{u}}\right)^{T}
$$

where $\boldsymbol{K}_{t}$ is the tangent stiffness matrix at the converged solution and the superscript, $T$, indicates the transpose of the matrix. The derivative of the residual vector with the design variable $\rho_{e}, \frac{\partial r}{\partial \rho_{e}}$, can be obtained using the chain rule approach. As seen in Eq. 12, the average engineering stresses are stated in terms of the internal force vector $f^{\text {int }}$. Hence the derivatives of the objective and constraints with respective to the displacement vector $\boldsymbol{u}$ can be written in terms of tangent stiffness matrix. See additional details in [34] and [35].

The optimization problem is solved using a flexible framework for large scale topology optimization based on PETSc [41, 42]. Using the sensitivities of the objective and constraints, $\frac{\partial \phi}{\partial \rho_{e}}$, the unit cell is iteratively updated using the Method of Moving Asymptotes (MMA) [48]. 


\section{Result}

In this section, the proposed optimization formulation is employed to design $3 \mathrm{D}$ auxetic materials for different prescribed Poisson's ratios under a tensile test. Three longitudinal strain points are considered to achieve constant Poisson's ratio in the strain interval of $[0.00,0.20]$, i.e. $\varepsilon \in\{0.001,0.10,0.20\}$. All the topology optimized designs are the results of topology optimization with a random initial distribution of design variables.

(a)

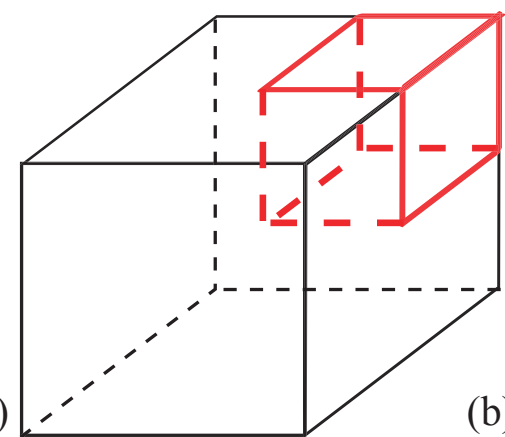

(b)

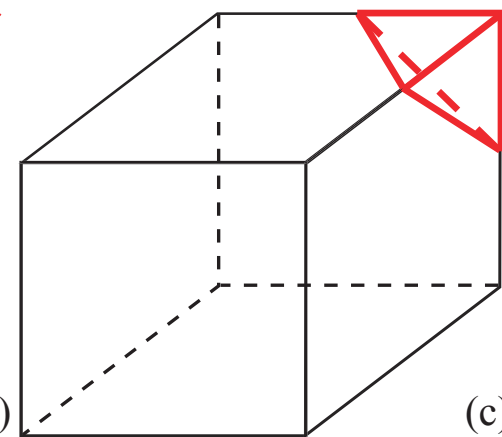

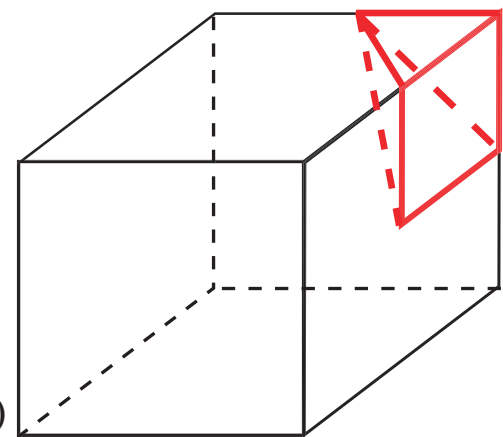

Figure 2: Illustration of the design domain in the unit cells with different symmetries. The design domains are represented by the regions enclosed by the red bold lines. (a) Design domain in the unit cell with plane symmetry. (b) Design domain in the unit cell with cubic symmetry. (c) Design domain in the unit cell with plane and rotational symmetry.

Design of 3D materials requires extensive computational power since the number of degrees of freedom explodes with the dimensionality. In order to obtain the optimized materials within reasonable time, the unit cell is discretized into $120 \times 120 \times 120$ elements. In this paper, all the topology optimized designs are obtained within 48 hours on 240 CPU cores with 280 optimization iterations. Due to the density filtering imposed by the PDE filter, the design is meshindependent. The reader is referred to Ref [46] for more details. To enhance the design robustness with respect to the manufacturing imperfections and ensure the length scale of the optimized auxetic materials, three design realizations have been considered in the optimization procedure by choosing three different thresholds of $\eta \in\{0.49,0.50,0.51\}$ in Eq. (14) to mimic the dilated, ideal and eroded manufacturing process, respectively. The objective function is extended to minimize the maximum lateral stress among the three different design realizations in the target strain interval, which guarantees the optimized designs perform well under the manufacturing tolerance considered. In this paper, a filter radius of $r=0.1 / 2 / \sqrt{3}$ of the cell size is used in the PDE filter. It corresponds to a minimal length scale of 0.02 in both the base material and void regions according to the length scale calculation in Ref [31]. To avoid confusion, only the blueprint design with $\eta=0.5$ is presented in the following sections, unless otherwise stated. The volume fraction is set to $10 \%$ to achieve lightweight lattice materials. The material properties of the base material are set to $E_{1}=1$ and $v=0.4$, respectively. 
Three different design symmetries are considered in this paper, i.e. plane symmetry in the three principal axes, cubic symmetry, and plane and rotational symmetry. A unit cell in Fig. 11 with plane symmetry is symmetric along all the three middle planes. In the unit cell with plane symmetry, the design variables in the two elements located at $\left(x_{1}, y_{1}, z_{1}\right)$ and $\left(1-x_{1}, y_{1}, z_{1}\right)$, respectively, are same. Likewise, the elements located at $\left(x_{1}, y_{1}, z_{1}\right)$ and $\left(x_{1}, 1-y_{1}, z_{1}\right)$ have the same design variable and the ones located at $\left(x_{1}, y_{1}, z_{1}\right)$ and $\left(x_{1}, y_{1}, 1-z_{1}\right)$ have the same design variable. Hence the design domain is one eighth of the unit cell as indicated by the region enclosed by the red lines in Fig. 22(a). In a unit cell with cubic symmetry, the three elements located at $\left(x_{1}, y_{1}, z_{1}\right),\left(y_{1}, z_{1}, x_{1}\right)$ and $\left(z_{1}, x_{1}, y_{1}\right)$ have the same design variable, and the elements located at $\left(x_{1}, y_{1}, z_{1}\right)$ and $\left(y_{1}, x_{1}, z_{1}\right)$ have the same design variable, beside plane symmetry. With the cubic symmetry, only one forty-eighth of the unit cell makes up the design domain, as shown in Fig. 2 (b), highlighted by the red bold lines. The plane and rotational symmetry is obtained by relaxing the cubic symmetry, such that design variables in the elements located at $\left(x_{1}, y_{1}, z_{1}\right)$ and $\left(y_{1}, x_{1}, z_{1}\right)$ are different. With the plane and rotational symmetry, only one twenty-fourth of the unit cell makes up the design domain as seen in Fig. 22(c).

\subsection{D optimized linear and nonlinear auxetic material with plane symmetry}

In the first case, the performance of auxetic materials considering small and finite deformations are compared. The material unit cells are assumed to be plane symmetry. The design domain is illustrated in Fig. 2(a). In this case, the tensile tests are performed along the three principal directions in the optimization process and the target Poisson's ratio is set to $v^{*}=-0.5$.

Fig 3 presents the optimized material unit cells considering small and finite deformations. The discreteness of the optimized designs is measured by a gray level indicator, defined as $M_{n d}=4(\mathbf{1}-\overline{\boldsymbol{\rho}})^{T} \overline{\boldsymbol{\rho}} \times 100 \%$. For the optimized unit cells, the gray level indicator is smaller than $2 \%$.

Fig 3 (a) - (d) present the optimized material unit cell under small deformations and corresponding performance. Fig 3 (a) shows the optimized material configuration obtained by removing all the elements with physical densities below 0.5 , where stair casing is observed due to the discretization. The stair-case-representation can be smoothed in the post process as illustrated in Fig 3 (b). The smoothed unit cell can be directly used for 3D printing. Fig. 3 (c) presents the slice view of the optimized unit cell in the middle plane perpendicular to the $x$-axis. It is seen that the optimized material possesses a length scale in both solid and void phases and hence no small features are observed. Fig. 3 (d) shows the performance of the optimized material considering finite strains. The optimized material shows nonlinear Poisson's ratio profile in the strain interval of $\varepsilon \in[0.00,0.20]$. Poisson's ratio is seen to grow with increased strain. The performance of the optimized material is evaluated at eleven equidistant strain points between $\varepsilon=0.001$ and $\varepsilon=0.20$. 

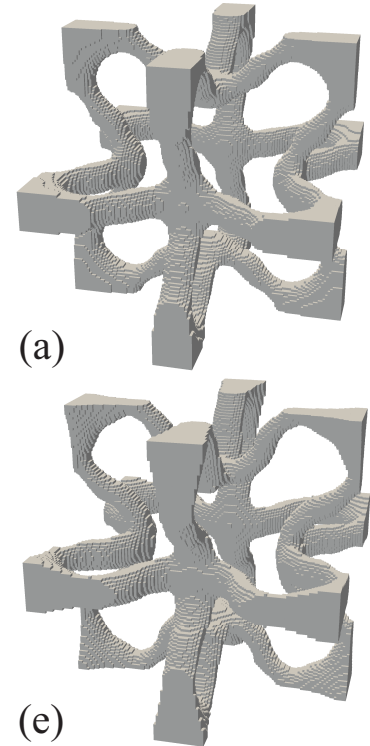

(b)

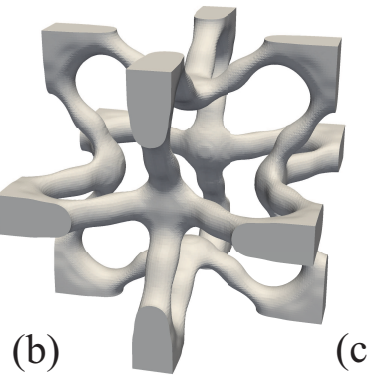

(c)
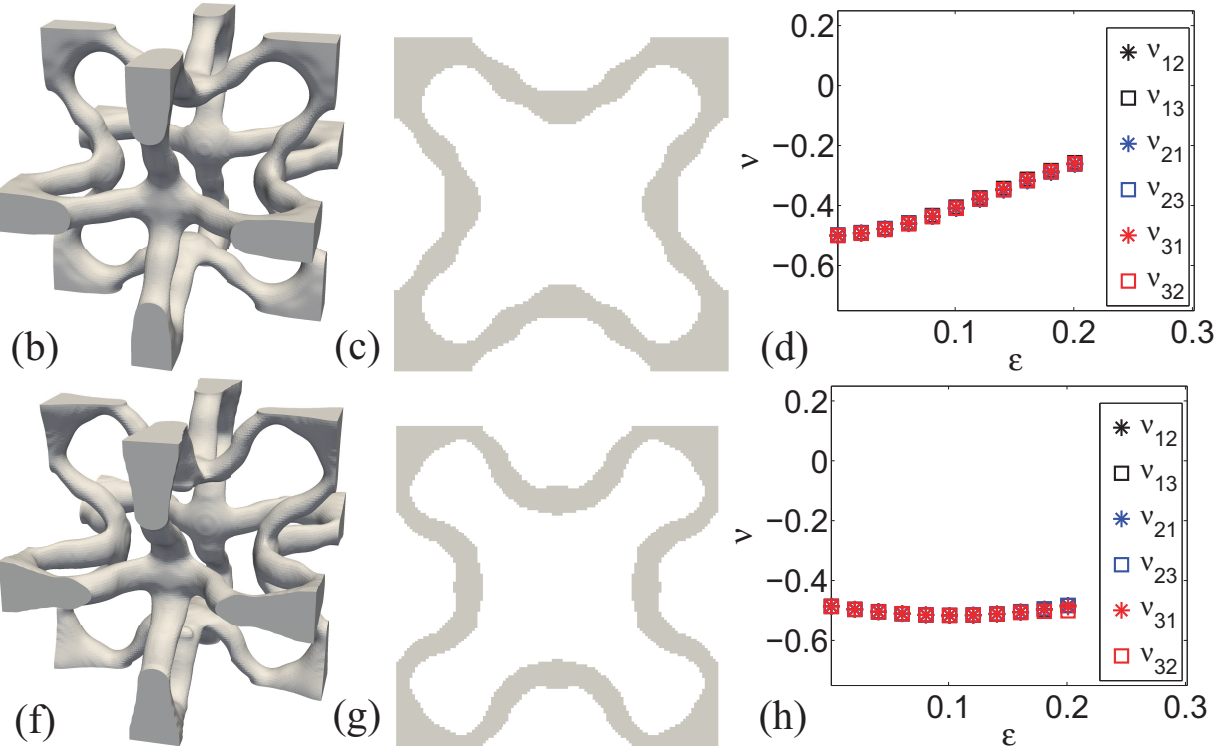

Figure 3: 3D optimized material configuration for $v^{*}=-0.5$ under small and finite deformations. Top row: Optimized material configuration accounting for small deformations and corresponding performance. Bottom row: Optimized material configuration accounting for finite deformations and corresponding performance. (a) Optimized material configuration representation with a threshold of $\eta=0.5$; (b) Smoothed optimized material configuration using iso-volume threshold. (c) Slice view of the optimized material at $x=0.5$; (d) Performance of the optimized material under uniaxial tensile tests along the three axes. (e) Optimized material configuration representation; (f) Smoothed optimized material configuration. (g) Slice view of the optimized material. (h) Performance of the optimized material under uniaxial tensile tests along the three principal directions.

Fig. 3 (e) - (h) show the optimized material unit cell accounting for finite strains. It is noticed that the optimized material has the same topology as the linear material in Fig. 3 (a) - (d). However, the optimized nonlinear material exhibits arch shapes with larger slope than the linear one as seen by comparing the slice views. Negative Poisson's ratio is induced by the expansion of the arch regions under stretching. The nonlinear material exhibits almost constant Poisson's ratio in the design strain interval of $\varepsilon \in[0.00,0.20]$, as seen in Fig. 3 (h).

The difference in Poisson's ratio behavior between the linear and nonlinear optimized materials can be further verified by investigating the deformed material configurations under different uniaxial strains as shown in Fig. 4. Under a small longitudinal strain, the linear material expands laterally as the nonlinear material. However, the lateral expansion in the linear material starts to saturate under a certain longitudinal strain because the arch shape becomes much flatter. This leads to a larger Poisson's ratio exactly as shown in Fig. 3 In comparison, the nonlinear material still retains the arch shape for the longitudinal strain of 0.20 even though its arch gets shallow, and hence exhibits a constant Poisson's ratio of $v^{*}=-0.5$ over the whole design strain range. 


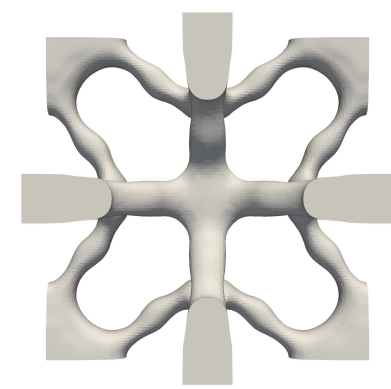

$\varepsilon=0.00$

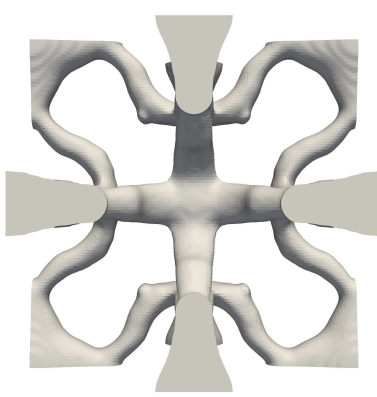

$\varepsilon=0.00$

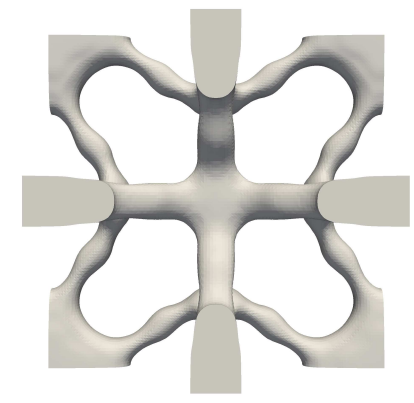

$\varepsilon=0.02$

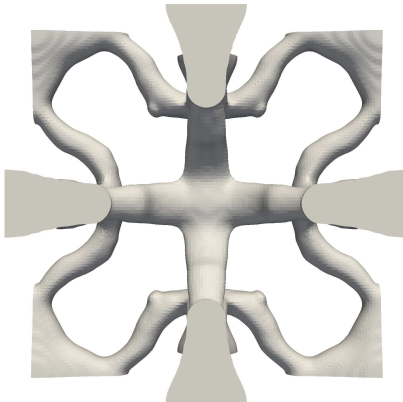

$\varepsilon=0.02$

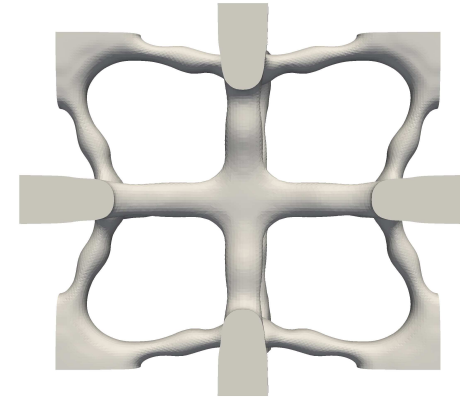

$\varepsilon=0.20$

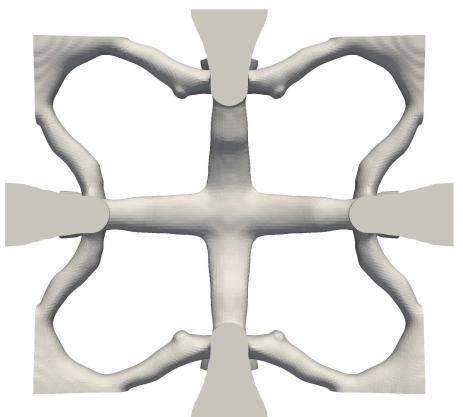

$\varepsilon=0.20$

Figure 4: Deformation of the optimized unit cell under different uniaxial strains. Top row: Unit cell optimized using small deformation theory. Bottom row: Unit cell optimized considering finite strains. Left column: undeformed unit cell with $\varepsilon=0.00$, Middle column: $\varepsilon=0.02$, Right column: $\varepsilon=0.20$,

\subsection{D auxetic nonlinear material with cubic symmetry}

It is evident from the previous section that the optimized nonlinear materials exhibit almost identical features along all the three principal axes and only small deviation is observed. This indicates that we can enforce cubic symmetry without sacrificing the performance of the optimized materials. With the cubic symmetry, the optimized materials exhibit identical performance along all the three principal axes. Moreover, the Poisson's ratios in both lateral directions are equal, i.e. $v_{12}=v_{13}$ and the averaged lateral stresses are also equal, i.e. $\bar{\sigma}_{22}^{1}=\bar{\sigma}_{33}^{1}$. With the cubic symmetry, only the tensile test along the $x$-axis is evaluated and the objectives are set to $\bar{\sigma}_{22}^{1}$ for different target strain points considered in the optimization procedure. Hence, the computational cost is reduced to be less than one third of the previous case. The design domain of a unit cell with cubic symmetry is illustrated in Fig. 2(b).

Materials are optimized for different prescribed Poisson's ratio of $v^{*} \in\{-0.2,-0.4,-0.6,-0.8\}$. The optimized material configurations are summarized in Fig. 5. It is seen that the optimized materials share the same topology as the materials presented in the previous section. The slice views of the optimized materials demonstrate that the arch shape get deeper as the target Poisson's ratio gets more negative. This indicates the nonlinear material with more negative Poisson's ratio expands more than the one with higher Poisson's ratio for a given uniaxial stretching. The 

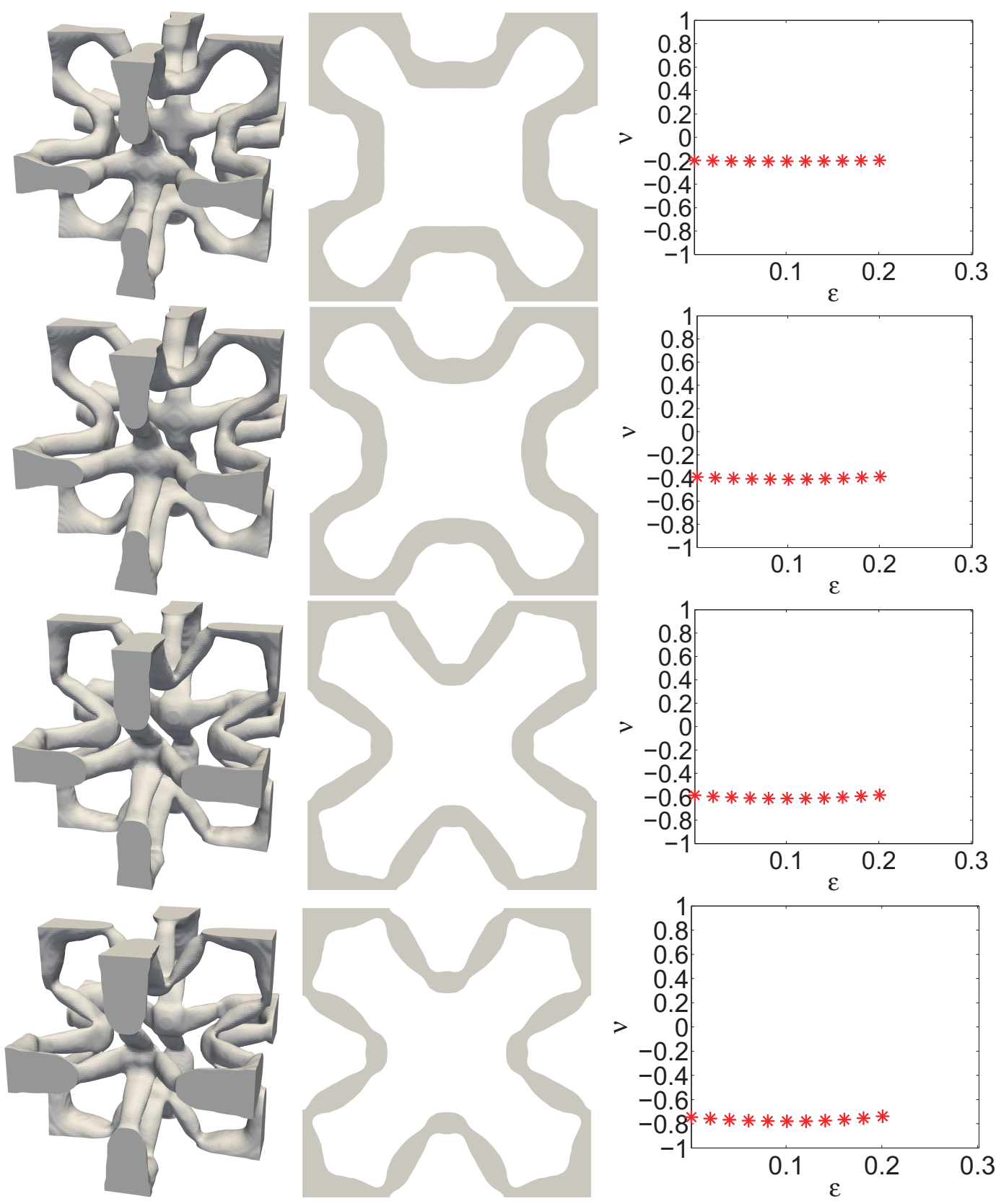

Figure 5: 3D optimized nonlinear material configurations with cubic symmetry under finite strains for $v^{*}=-0.2, v^{*}=-0.4$, $v^{*}=-0.6$ and $v^{*}=-0.8$ and corresponding performance in the target strain interval. Left column: 3D view of the optimized nonlinear materials. Middle column: Slice view at the middle plane. Right column: Poisson's ratio profiles of the optimized nonlinear materials in the target strain interval of $\varepsilon \in[0.00,0.20]$.

length scale is ensured by the robust formulation as shown in 3D and slice views of the optimized nonlinear materials. No small details are observed in the optimized materials. The optimized material for $v^{*}=-0.8$ exhibits slightly bigger variations in terms of actual Poisson's ratios in the target strain region, compared to the one for $v^{*}=-0.2$. However, 
the variation of Poisson's ratio is much smaller compared to the linear material in the previous section.

In order to quantitatively evaluate the performance of the optimized auxetic materials, the mean value of the Poisson's ratios $\bar{v}$ and the standard deviation of the Poisson's ratio, SD, are measured in the target strain range, defined as,

$$
\bar{v}=\sum_{m=1}^{N}\left(v_{12}\left(\varepsilon^{1 m}\right)+v_{13}\left(\varepsilon^{1 m}\right)\right) / 2 N, \quad \mathrm{SD}=\sqrt{\sum_{m=1}^{N}\left(\left(\left(v_{12}\left(\boldsymbol{\varepsilon}^{1 m}\right)-\bar{v}\right) / 2 N\right)^{2}+\left(\left(v_{13}\left(\boldsymbol{\varepsilon}^{1 m}\right)-\bar{v}\right) / 2 N\right)^{2}\right)}
$$

where $N=11$ is the total number of the evaluation strain points in the strain interval of $\varepsilon \in[0.001,0.20]$.

The performances of the optimized auxetic materials are summarized in Tab. 1] It is seen that the averaged Poisson's ratios are very close to the target Poisson's ratio except the one for $v^{*}=-0.8$ where the averaged Poisson's ratio is larger than the prescribed one. In all the cases, the standard deviation is smaller than 0.0042 . This implies that all the auxetic lattice materials exhibit a very small variation of Poisson's ratio in the target strain interval.

\begin{tabular}{ccc}
\hline$v^{*}$ & $\bar{v}$ for $\varepsilon \in[0.00,0.20]$ & $\mathrm{SD}$ \\
\hline-0.8 & -0.7650 & 0.0041 \\
-0.6 & -0.6036 & 0.0031 \\
-0.4 & -0.4025 & 0.0024 \\
-0.2 & -0.2009 & 0.0009 \\
\hline
\end{tabular}

Table 1: Quantitative evaluations of the optimized auxetic materials with cubic symmetry in the target strain interval of $\varepsilon \in$ $[0.00,0.20]$

Fig. 6 shows the performance of the optimized materials of $v^{*}=-0.6$ under the manufacturing imperfections considered in this paper mimicking by the thresholds of $\eta \in\{0.49,0.50,0.51\}$. The left panel in Fig. 6 illustrates the manufacturing imperfections using the contour plot of three different design realizations in the slice view at the middle plane, where the black solid line represents the blueprint with $\eta=0.50$. It is seen that the three design realizations share the same topology and that the blueprint design possesses length scales in both phases. The performance of the optimized material under the manufacturing imperfections is shown in the right panel. It is remarked that under the considered manufacturing imperfections, the optimized material preserves an averaged Poisson's ratio of $\bar{v}=-0.6042$ with a bigger standard deviation of 0.0050 compared with the performance of the blueprint design with $\eta=0.50$.

\subsection{Nonlinear material with plane and rotational symmetry}

In this section, the cubic symmetry is relaxed and only plane and rotational symmetry along all the three principal axes is imposed. The rotational symmetry ensures that elements located at $(x, y, z),(y, z, x)$ and $(z, x, y)$ are identical. The computational cost of evaluating the Poisson's ratios is the same as the previous section since only the tensile 

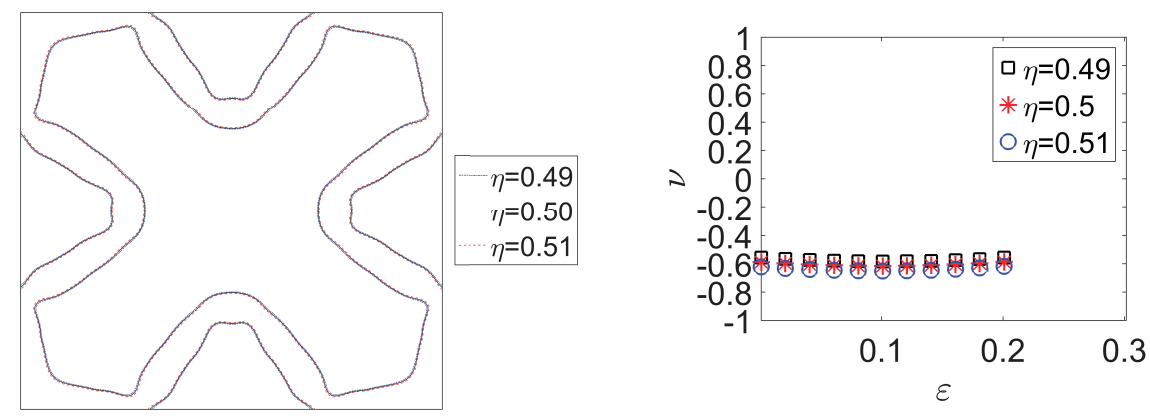

Figure 6: Performance of the optimized nonlinear material with cubic symmetry for $v^{*}=-0.6$ under manufacturing imperfections considered. Left: Illustration of manufacturing imperfections considered in the optimization procedure using contour plots in the slice view at the middle plane with $\eta \in\{0.49,0.50,0.51\}$. Right: Performance of the optimized material under the manufacturing imperfections.

tests in one principle direction is considered. The design domain is sketched in the unit cell in Fig. 2 (c), i.e. the region enclosed by the red bold lines.

Under such a symmetry condition, neither the Poisson's ratios nor the averaged lateral stresses in the two lateral directions are equal. Hence both the averaged lateral engineering stresses, $\bar{\sigma}_{22}^{1}$ and $\bar{\sigma}_{33}^{1}$, are considered in the optimization process. Fig. 7 summarizes the optimized nonlinear materials for different target Poisson's ratios and corresponding performances. The optimized materials consisting of $2 \times 2 \times 2$ unit cells are presented in the middle row in Fig. 7 to illustrate how the optimized unit cells tessellate in 3D, where the basic cells are highlighted in the copper-like color. The optimized materials of $v^{*}=-0.2, v^{*}=-0.4$ and $v^{*}=-0.6$ exhibit the same topology and the optimized material of $v^{*}=-0.8$ displays a different topology. In all the optimized material configurations, tilted members intersect and form members along the principal directions. Length scales are observed in all the optimized materials.

Closer inspections show that the negative Poisson's ratios result from a chiral mechanism [16]. Under a uniaxial stretching, the flat members along the other two directions move towards each other. This leads to expansions in these two directions and results in negative Poisson's ratios. The deformed optimized material configurations shown in Fig. 8 further demonstrate the mechanisms which lead to auxetic behaviour. In the optimized material of $v^{*}=-0.8$, the flat members in the out-of-plane direction come closer to each other and lead to a larger expansion of the flat members in the vertical direction. Hence the optimized material exhibits much lower Poisson's ratio compared to the one obtained for $v^{*}=-0.4$

The mean and standard deviation of the performances for the optimized auxetic materials are summarized in Tab.

2. The deviation of the Poisson's ratios with respect to the average Poisson's ratio in this case is still very small and less than 0.0031 . 

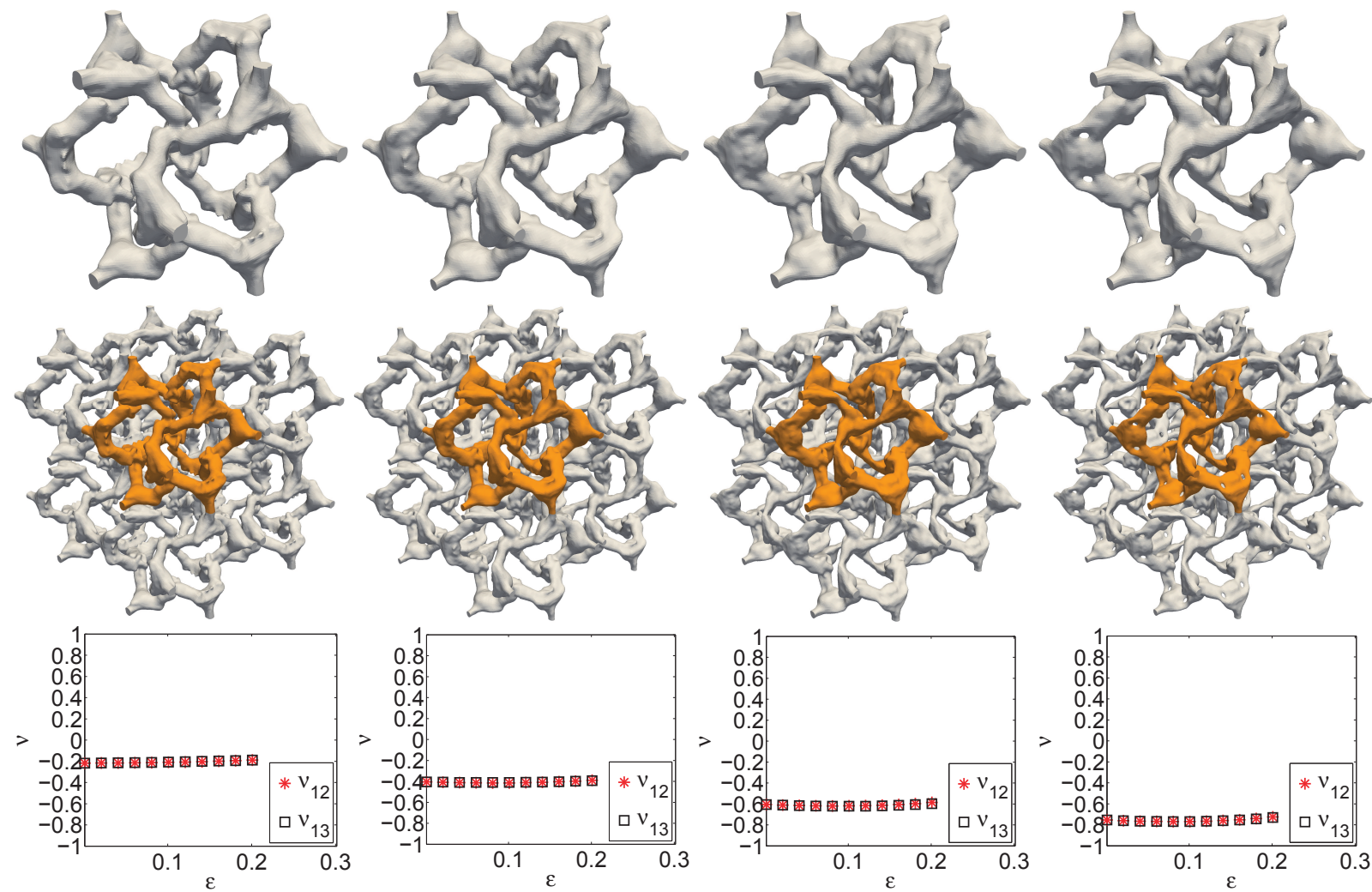

Figure 7: Optimized auxetic lattice materials with plane and rotational symmetry and corresponding performances for $v^{*}=-0.2$, $v^{*}=-0.4, v^{*}=-0.6$ and $v^{*}=-0.8$ from the left column to the right one. Top row: Optimized material unit cells. Middle row: View of the optimized materials with $2 \times 2 \times 2$ unit cells, where the basic unit cells are highlighted in the copper-like color. Bottom row: Poisson's ratio profile of the optimized materials in the target strain interval.

\begin{tabular}{ccc}
\hline$v^{*}$ & $\bar{v}$ for $\varepsilon \in[0.00,0.2]$ & $\mathrm{SD}$ \\
\hline-0.8 & -0.7578 & 0.0027 \\
-0.6 & -0.6117 & 0.0030 \\
-0.4 & -0.4066 & 0.0015 \\
-0.2 & -0.2068 & 0.0020 \\
\hline
\end{tabular}

Table 2: Performances of the optimized auxetic material configurations with plane and rotational symmetry in the target strain interval of $\varepsilon \in[0.00,0.20]$

\subsection{Material architecture interpolation based on subsequent simplification and shape optimization}

The topology optimized materials with cubic symmetry in Fig. 5 exhibit identical topology for different target Poisson's ratios. It opens the possibility to interpolate the material architecture as functions of Poisson's ratio as done for the 2D case by Clausen et al [36]. This section aims at interpolating material architectures as functions of Poisson's ratios based on shape-optimized auxetic materials with fixed cylindric member size, such that one can obtain the material architecture for any given Poisson's ratio.

Inspired by topology optimized material architectures in Fig. 5, we can parametrize auxetic materials using 


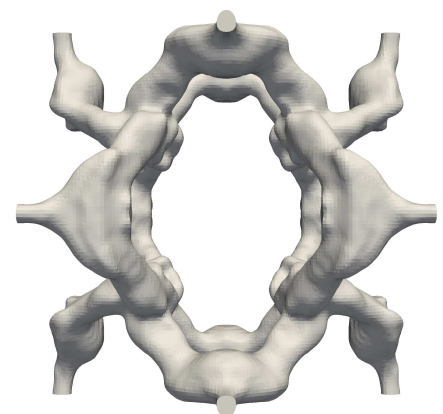

$\varepsilon=0.00$

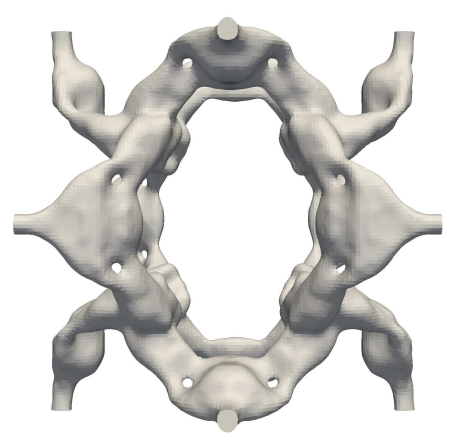

$\varepsilon=0.00$

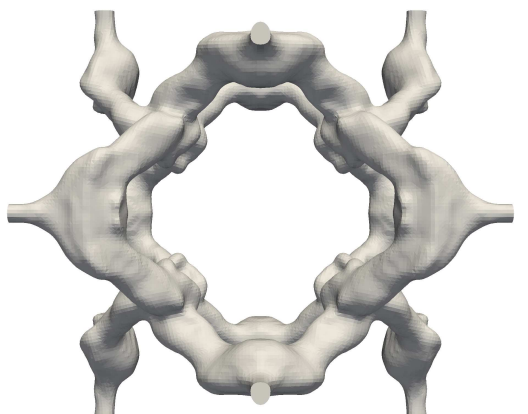

$\varepsilon=0.20$

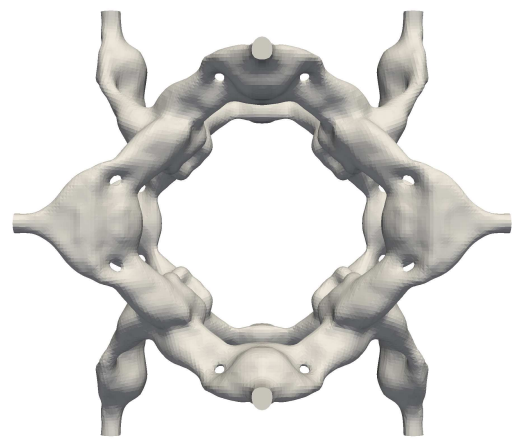

$\varepsilon=0.20$

Figure 8: Deformation of the optimized materials under the uniaxial stretching. Top row: Optimized material for $v^{*}=-0.4$. Bottom row: Optimized material for $v^{*}=-0.8$. Left column: Top view of the undeformed unit cell. Right column: Top view of the deformed unit cell for $\varepsilon=0.20$.

cylinders with a fixed radius, controlled by several points, as illustrated in Fig. 9 By designing the control points, we can optimize the auxetic material architectures to achieve the target Poisson's ratio $v^{*}$.

The cylinder controlled by the $i$ th and $(i+1)$ th points located at $\boldsymbol{y}_{i}=\left(y_{i}^{1}, y_{i}^{2}, y_{i}^{3}\right)$ and $\boldsymbol{y}_{i+1}=\left(y_{i+1}^{1}, y_{i+1}^{2}, y_{i+1}^{3}\right)$ can be approximated by a super-ellipsoid, such that it is differentiable with respect to the control points. Here superscripts represent the axial directions. The center of the cylinder is defined as $\boldsymbol{c}_{i}=\left(\boldsymbol{y}_{i}+\boldsymbol{y}_{i+1}\right) / 2$. The super-ellipsoid is defined by

$$
H\left(\boldsymbol{x}, \boldsymbol{y}_{i}, \boldsymbol{y}_{i+1}\right)=\frac{\|a\|^{2}}{r^{2}}+\frac{\|b\|^{2}}{r^{2}}+\frac{\|c\|^{100}}{l_{i}^{100}}-1
$$

where $\boldsymbol{x}=\left(x^{1}, x^{2}, x^{3}\right)$ represents any given location, $r$ is the prescribed radius of the cylinder and is set to $r=0.03$ and $l_{i}$ is the half distance between two points defined by $l_{i}=\left\|\boldsymbol{y}_{i+1}-\boldsymbol{y}_{i}\right\| / 2$, the corresponding exponent is chosen as 
100 to approximate a cylinder, and the parameters $a, b$ and $c$ are defined by

$$
\begin{aligned}
& a=\left(x^{1}-\left(y_{i+1}^{1}+y_{i}^{1}\right) / 2\right) \cos \left(\theta_{1}\right)-\left(x^{2}-\left(y_{i+1}^{2}+y_{i}^{2}\right) / 2\right) \sin \left(\theta_{1}\right) \\
& b=\left(\left(x^{1}-\left(y_{i+1}^{1}+y_{i}^{1}\right) / 2\right) \sin \left(\theta_{1}\right)+\left(x^{2}-\left(y_{i+1}^{2}+y_{i}^{2}\right) / 2\right) \cos \left(\theta_{1}\right)\right) \cos \left(\theta_{2}\right)-\left(x^{3}-\left(y_{i+1}^{3}+y_{i}^{3}\right) / 2\right) \sin \left(\theta_{2}\right) \\
& c=\left(\left(x^{1}-\left(y_{i+1}^{1}+y_{i}^{1}\right) / 2\right) \sin \left(\theta_{1}\right)+\left(x^{2}-\left(y_{i+1}^{2}+y_{i}^{2}\right) / 2\right) \cos \left(\theta_{1}\right)\right) \sin \left(\theta_{2}\right)+\left(x^{3}-\left(y_{i+1}^{3}+y_{i}^{3}\right) / 2\right) \cos \left(\theta_{2}\right)
\end{aligned}
$$

Here $\theta_{1}$ and $\theta_{2}$ denote the angle of the vector $\boldsymbol{y}_{i+1}-\boldsymbol{y}_{i}$ with respect to the $x$-axis in the $x$ - $y$ plane and the one between the vector and the $x-y$ plane, respectively. If the location $\boldsymbol{x}$ is located inside the $i$ th super-ellipsoid, $H\left(\boldsymbol{x}, \boldsymbol{y}_{i}, \boldsymbol{y}_{i+1}\right)<0$ and if outside, $H\left(\boldsymbol{x}, \boldsymbol{y}_{i}, \boldsymbol{y}_{i+1}\right)>0$.

The geometry of a unit cell can be represented by several super-ellipsoids. For any given super-ellipsoid, the region inside the super-ellipsoid is assigned to be the base material and the region outside is assigned to be void. The final physical density of element $e$ resulting from all the $q-1$ super-ellipsoids controlled by $q$ points is calculated by

$$
\bar{\rho}_{e}=1-\Pi_{i=1}^{q-1} \frac{1}{1+\exp \left(-\beta_{2} H\left(\boldsymbol{x}_{e}, \boldsymbol{y}_{i}, \boldsymbol{y}_{i+1}\right)\right)}
$$

where $\boldsymbol{x}_{e}$ is the centroid of element $e$ and $\beta_{2}$ is set to $\beta_{2}=10$.

To smooth the intersection between two super-ellipsoids, the PDE filter [46] is employed with $r=0.1 / 6.0 / \sqrt{3}$ together with the projection in Eq. 14 with $\beta=16$. The sensitivities of the physical density $\bar{\rho}_{e}$ with respect to design geometrical parameters $\boldsymbol{y}_{i}$ can be analytically derived using Eq. (20)-(24). The sensitivities of objective and constraints with respect to design variables are calculated using the chain rule approach in [35]. Using the sensitivities, the unit cell configuration is iteratively updated using MMA [48].

In the following, auxetic materials with cubic symmetry are designed as in subsection 3.2 The material configurations are parametrized using seventy-two super-ellipsoids controlled by sixty four points indicated by the asterisks in the middle panel of Fig. 9. Due to the imposed symmetry, the total points are projected using four designable control points at the middle plane perpendicular to the $z$-axis, indicated with red circles in the left panel of Fig. 9 To simplify the material configuration interpolation later, the design parameters are set to be $x$-coordinate of the second point as well as $y$-coordinate of the third and fourth points (see Fig. 97. Hence the four designable control points are set to $\boldsymbol{y}_{1}=(0.0,0.0,0.5), \boldsymbol{y}_{2}=(x, 0.0,0.5), \boldsymbol{y}_{3}=(0.45, y, 0.5)$ and $\boldsymbol{y}_{4}=(0.5, y, 0.5)$. The right panel in Fig. 9 shows the corresponding material configuration.

The shape optimized material configurations and corresponding mean value and standard deviation for different target Poisson's ratios are summarized in Tab. 3. It is seen that the optimized materials exhibit constant Poisson's 

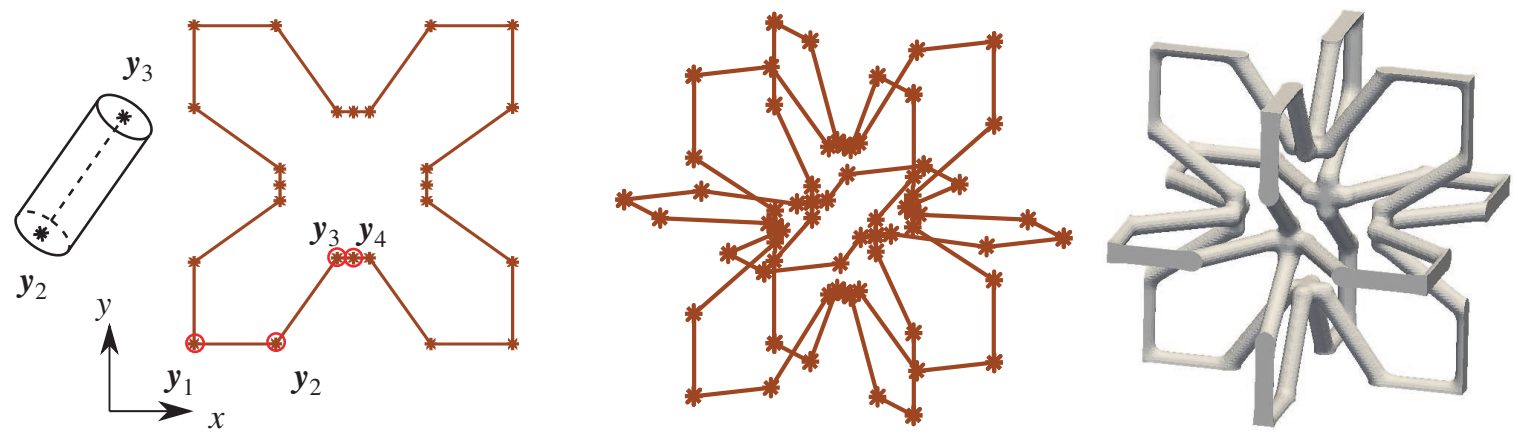

Figure 9: Illustration of the super-ellipsoid parametrization of the auxetic material architecture. Left: Slice view of super-ellipsoid parametrization of the auxetic material architecture at the middle plane perpendicular to the $z$-axis. Asterisks represent the control points and the red circles represent the designable control points according to the unit cell symmetry. Middle: Super-ellipsoid parametrization of the auxetic material architecture with asterisks representing the control points. Right: Auxetic material architecture generated using the super-ellipsoid parametrization.

\begin{tabular}{ccccc}
\hline$v^{*}$ & $\bar{v}$ for $\varepsilon \in[0.00,0.20]$ & SD & $x$ & $y$ \\
\hline-0.8 & -0.7800 & 0.0031 & 0.1806 & 0.3830 \\
-0.6 & -0.5990 & 0.0044 & 0.2570 & 0.2702 \\
-0.4 & -0.4030 & 0.0044 & 0.3019 & 0.2075 \\
-0.2 & -0.1991 & 0.0023 & 0.3435 & 0.1492 \\
0.0 & -0.00185 & 0.0004 & 0.4000 & 0.0700 \\
\hline
\end{tabular}

Table 3: Summary of the shape optimized auxetic material configurations and corresponding performances in the target strain interval of $\varepsilon \in[0.00,0.20]$
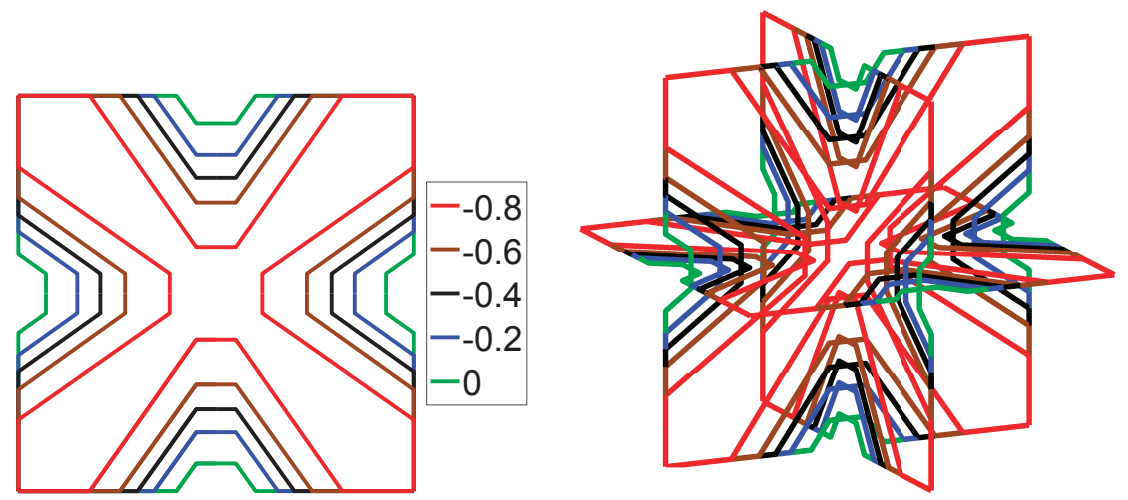

Figure 10: Skeleton views of shape optimized materials for different target Poisson's ratios. Left: Slice view. Right: 3D view.

ratios in the target strain interval and the deviation of Poisson's ratios is smaller than 0.0045 . The skeleton views of the optimized material architectures are presented in Fig. 10 .

By interpolating the five optimized material configurations, the material architectures for any given Poisson's ratio in the interval of $[-0.78,0.00]$ are obtained. Here we use quadratic functions to interpolate the design parameters, $x$ and $y$. To get more accurate interpolation, the averaged Poisson's ratios are used instead of the prescribed ones in Tab. 
3 in the interpolation function. The final expressions of $x$ and $y$ are presented in Eq. 25].

$$
\begin{aligned}
& \xi=\frac{\left(v^{*}+0.3993\right)}{0.3104} \\
& x=-0.0002242 \xi^{4}+0.01652 \xi^{3}-0.008549 \xi^{2}+0.06087 \xi+0.3034 \\
& y=0.00208 \xi^{4}-0.02523 \xi^{3}+0.01094 \xi^{2}-0.08428 \xi+0.2054
\end{aligned}
$$

where $v^{*}$ represents a given Poisson's ratio.

The performances of the interpolated materials are evaluated for forty given Poisson's ratios, equally distributed in the Poisson's ratio interval $v^{*} \in[-0.78,0.00]$. The performances are summarized in Fig. 11. The average Poisson's ratios deviate slightly from the given ones indicated by the dashed line in the left panel in Fig. 11 . However, the standard deviations of the interpolated material architectures are small. This indicates that all interpolated materials exhibit constant Poisson's ratios.
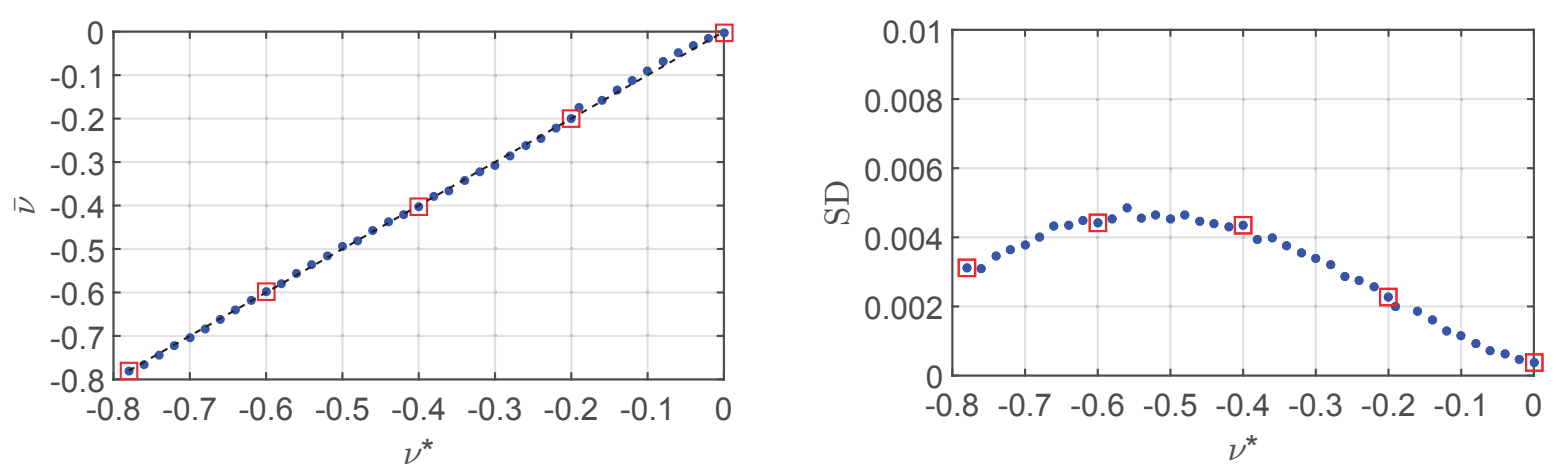

Figure 11: Average Poisson's ratios and corresponding standard deviations of the interpolated materials for any given Poissons ratio in $v^{*} \in[-0.78,0.00]$. The red square points indicate the five optimized reference structures.

\section{Discussion}

Systematic experimental verifications of the optimized materials are out of the scope of this paper and will be performed in a future study. However, in order to demonstrate the physical performance of a material optimized for negative Possion's ratio, the optimized material with cubic symmetry for $v^{*}=-0.6$ in Fig. 5 was fabricated. The material was fabricated using a desktop stereolithography (SLA) 3D Printer from Formlabs, i.e. Form 2 [50] using rubber [51]. Due to the geometrical complexity of the optimized material, a lot of supports are needed for successful fabrication, which for the 3D printer employed was impossible to remove. Hence the material block was printed as four separate samples consisting of $6 \times 4 \times 1$ unit cells with each unit cell of $2 \mathrm{~cm} \times 2 \mathrm{~cm} \times 2 \mathrm{~cm}$ and then manually glued together to form a sample consisting of $6 \times 4 \times 4$ unit cells as shown in the left panel in Fig 12 The sample 
was fabricated by Jesper Baunsøe Nielsen at the Department of Mechanical Engineering in Technical University of Denmark. By compressing the sample, the deformed sample clearly shows a negative Poisson's ratio as can be seen in the middle and right panels in Fig. 12. Due to the few cell repetitions, accurate determination of the effective Poisson's ratio is not attainable.
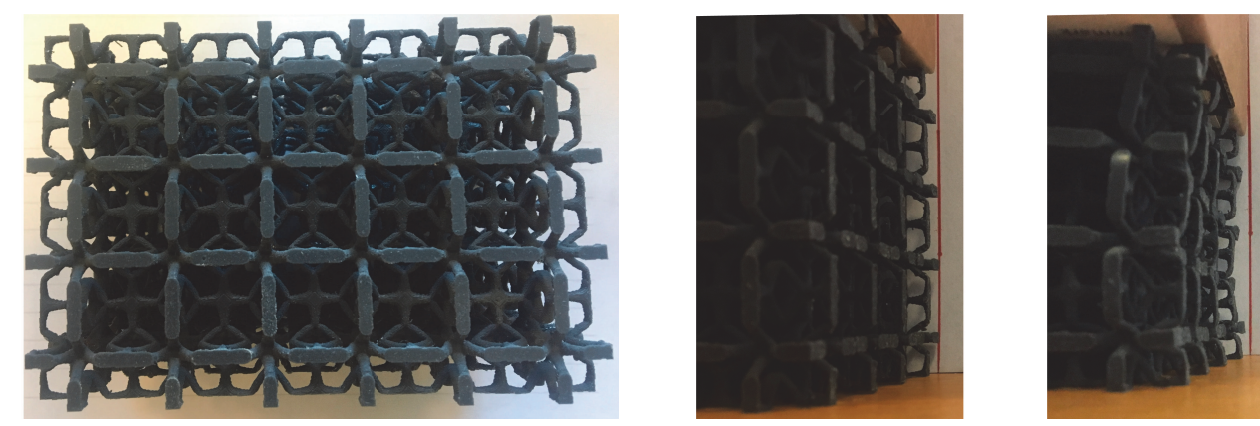

Figure 12: Fabricated sample of the optimized material using 3D printing and demonstration of the performance of the fabricated sample under compression. Left: Fabricated sample. Middle: Undeformed sample. Right: Deformation of the sample under compression.

In the future study on the experimental verification of the optimized materials, samples with more cell repetitions, e.g. $13 \times 9 \times 9$ unit cells will be fabricated. In order to get rid of the boundary effect in the tensile test, the deformation of the center cell in the sample could be assessed by X-ray computed tomography (CT) scans. And the Poisson's ratio of the optimized material can be extracted using the cell deformation.

\section{Conclusion}

In the paper, 3D auxetic lattice materials are systematically designed for finite strains. A unit cell model has been established to mimic the uniaxial compression and tensile tests. The secant Poisson's ratios and secant stiffness can be evaluated using the proposed model. Based on the fact that the lateral engineering stresses on the faces parallel to the loading directions are equal to zero in the tests, the optimization problem for tailoring the Poisson's ratio profile of materials under finite strains has been formulation to minimize the average lateral engineering stresses under the prescribed deformations obtained using a target Poisson's ratio in a design strain interval.

3D auxetic materials have been systematically optimized for different target Poisson's ratios in a target strain interval of $\varepsilon \in[0.00,0.20]$ based on the proposed optimization formulation. By imposing two different symmetry conditions, two sets of material configurations have been obtained. Numerical results demonstrate that the lattice material architectures can be tuned to achieve different target Poisson's ratios in the tensile test. Furthermore, it is worthwhile to mention that the presented optimization formulation can be directly employed for material design in the compression test by prescribing $\varepsilon<0$. 
Inspired by the topology optimized material architectures, a subsequent shape optimization is proposed by parametrizing material configurations using a set of super-ellipsoids controlled by several control points. The configuration of simplified auxetic lattice materials are optimized by tuning two geometrical parameters in the control points for five different Poisson's ratios. By interpolating the five optimized material configurations, lattice material architectures are presented explicitly as functions of Poisson's ratios. Numerical evaluations show that all the interpolated materials possess constant Poisson's ratios in the target strain interval with very small deviation.

\section{Acknowledgments}

This work was financially supported by an ERC Starting Grant (INNODYN), and by Villum Fonden through the NextTop project. The author thanks Prof. Jakob S. Jensen at the Department of Electrical Engineering, Prof. Ole Sigmund, Dr. Niels Aage and Dr. Boyan S. Lazarov at the Department of Mechanical Engineering in Technical University of Denmark, for discussions. The author thanks Jesper Baunsøe Nielsen at the Department of Mechanical Engineering in Technical University of Denmark for fabricating the optimized unit cell.

[1] R. Lakes, Foam structures with a negative Poisson's ratio, Science 235 (4792) (1987) 1038-1040.

[2] K. E. Evans, Auxetic polymers: a new range of materials, Endeavour 15 (4) (1991) 170-174.

[3] A. Safari, Development of piezoelectric composites for transducers, Journal De Physique III 4 (7) (1994) 1129-1149.

[4] F. Scarpa, F. C. Smith, Passive and MR fluid-coated auxetic PU foam - mechanical, acoustic, and electromagnetic properties, Journal of Intelligent Material Systems and Structures 15 (12) (2004) 973-979.

[5] G. Shvets, Y. A. Urzhumov, Electric and magnetic properties of sub-wavelength plasmonic crystals, Journal of Optics A-pure and Applied Optics 7 (2) (2005) S23-S31.

[6] R. E. Christiansen, O. Sigmund, Designing Meta Material Slabs Exhibiting Negative Refraction Using Topology Optimization, Structural and Multidisciplinary Optimization 54 (3) (2016) 469-482, ISSN 16151488, 1615147x.

[7] R. E. Christiansen, O. Sigmund, Experimental validation of systematically designed acoustic hyperbolic meta material slab exhibiting negative refraction, Applied Physics Letters 109 (10) (2016) 66-70, ISSN 10773118, 00036951.

[8] K. L. Alderson, A. P. Pickles, P. J. Neale, K. E. Evans, Auxetic polyethylene: the effect of a negative Poisson's ratio on hardness, Acta Metallurgica Et Materialia 42 (7) (1994) 2261-2266.

[9] K. L. Alderson, A. Fitzgerald, K. E. Evans, The strain dependent indentation resilience of auxetic microporous polyethylene, Journal of Materials Science 35 (16) (2000) 4039-4047.

[10] J. B. Choi, R. S. Lakes, Fracture toughness of re-entrant foam materials with a negative Poisson's ratio: experiment and analysis, International Journal of Fracture 80 (1) (1996) 73-83.

[11] C. P. Chen, R. S. Lakes, Micromechanical analysis of dynamic behavior of conventional and negative Poisson's ratio foams, Journal of Engineering Materials and Technology 118 (3) (1996) 285-288.

[12] F. Scarpa, J. R. Yates, L. G. Ciffo, S. Patsias, Dynamic crushing of auxetic open-cell polyurethane foam, Proceedings of the Institution of Mechanical Engineers Part C: Journal of Mechanical Engineering Science 216 (12) (2002) 1153-1156.

[13] F. Scarpa, L. G. Ciffo, J. R. Yates, Dynamic properties of high structural integrity auxetic open cell foam, Smart Materials \& Structures 13 (1) (2004) 49-56.

[14] R. Lakes, Advances in negative Poisson's ratio materials, Advanced Materials 5 (4) (1993) $293-296$. 
[15] Q. Liu, Literature Review: Materials with Negative Poisson's Ratios and Potential Applications to Aerospace and Defence, Tech. Rep., 2006.

[16] R. Lakes, Deformation mechanisms in negative Poisson's ratio materials: structural aspects, Journal of Materials Science 26 (9) (1991) $2287-2292$.

[17] K. L. Alderson, K. E. Evans, The fabrication of microporous polyethylene having a negative Poisson's ratio, Polymer 33 (20) (1992) 44354438.

[18] K. E. Evans, J. P. Donoghue, K. L. Alderson, The design, matching and manufacture of auxetic carbon fibre laminates, Journal of Composite Materials 38 (2) (2004) 95-106

[19] D. Prall, R. S. Lakes, Properties of a chiral honeycomb with a Poisson's ratio of -1., International Journal of Mechanical Sciences 39 (3) (1997) 305-314.

[20] R. S. Lakes, R. Witt, Making and characterizing negative Poisson's ratio materials, International Journal of Mechanical Engineering Education 30 (1) (2002) 50-58.

[21] U. D. Larsen, O. Sigmund, S. Bouwstra, Design and fabrication of compliant micromechanisms and structures with negative Poisson's ratio, Journal of Microelectromechanical Systems 6 (2) (1997) 99-106.

[22] M. P. Bendsøe, O. Sigmund, Topology optimization: theory, methods and applications, Springer, Berlin, 2003.

[23] O. Sigmund, Materials with prescribed constitutive parameters: an inverse homogenization problem, International Journal of Solids and Structures 31 (17) (1994) 2313-2329.

[24] O. Sigmund, Tailoring materials with prescribed elastic properties, Mechanics of Materials 20 (4) (1995) $351-368$.

[25] E. Andreassen, B. S. Lazarov, O. Sigmund, Design of manufacturable 3D extremal elastic microstructure, Mechanics of Materials 69 (1) (2014) $1-10$

[26] R. B. Haber, C. S. Jog, M. P. Bendsøe, A new approach to variable-topology shape design using a constraint on perimeter, Structural Optimization 11 (1) (1996) 1-12.

[27] B. Bourdin, Filters in topology optimization., International Journal for Numerical Methods in Engineering 55 (2001) $2143-2158$.

[28] T. E. Bruns, D. A. Tortorelli, Topology optimization of non-linear elastic structures and compliant mechanisms, Computer Methods In Applied Mechanics and Engineering 190 (26-27) (2001) 3443-3459.

[29] O. Sigmund, Morphology-based black and white filters for topology optimization, Structural and Multidisciplinary Optimization 33 (4-5) (2007) 401-424.

[30] O. Sigmund, Manufacturing tolerant topology optimization, Acta Mechanica Sinica 25 (2009) 227-239.

[31] F. Wang, B. S. Lazarov, O. Sigmund, On projection methods, convergence and robust formulations in topology optimization, Structural and Multidisciplinary Optimization 43 (6) (2011) 767-784, ISSN 16151488.

[32] C. W. Smith, R. J. Wootton, K. E. Evans, Interpretation of experimental data for Poisson's ratio of highly nonlinear materials, Experimental Mechanics 39 (4) (1999) 356-362.

[33] T. Buhl, C. B. W. Pedersen, O. Sigmund, Stiffness design of geometrically nonlinear structures using topology optimization, Structural and Multidisciplinary Optimization 19 (2) (2000) 93-104.

[34] F. Wang, B. S. Lazarov, O. Sigmund, J. S. Jensen, Interpolation scheme for fictitious domain techniques and topology optimization of finite strain elastic problems, Computer Methods in Applied Mechanics and Engineering 276 (2014) 453-472, ISSN 18792138, 00457825.

[35] F. Wang, O. Sigmund, J. S. Jensen, Design of materials with prescribed nonlinear properties, Journal of the Mechanics and Physics of Solids 69 (1) (2014) 156-174, ISSN 18734782, 00225096.

[36] A. Clausen, F. Wang, J. S. Jensen, O. Sigmund, J. A. Lewis, Topology Optimized Architectures with Programmable Poisson's Ratio over Large Deformations, Advanced Materials 27 (37) (2015) 5523-5527, ISSN 15213862, 09481907, 15214095, 09359648. 
[37] S. Babaee, J. Shim, J. C. Weaver, E. R. Chen, N. Patel, K. Bertoldi, 3D Soft Metamaterials with Negative Poisson's Ratio, Advanced Materials 25 (36) (2013) 5044-5049, ISSN 09359648, 15214095.

[38] X. Ren, J. Shen, A. Ghaedizadeh, H. Tian, Y. M. Xie, Experiments and parametric studies on 3D metallic auxetic metamaterials with tuneable mechanical properties, Smart Materials and Structures 24 (9) (2015) -, ISSN 1361665x, 09641726.

[39] H. H. Huang, B. L. Wong, Y. C. Chou, Design and properties of 3D-printed chiral auxetic metamaterials by reconfigurable connections, Physica Status Solidi (b) Basic Research (2016) -ISSN 15213951, 03701972.

[40] L. Cabras, M. Brun, A class of auxetic three-dimensional lattices, Journal of the Mechanics and Physics of Solids 91 (2016) 56-72.

[41] S. Balay, S. Abhyankar, M. F. Adams, J. Brown, P. Brune, K. Buschelman, L. Dalcin, V. Eijkhout, W. D. Gropp, D. Kaushik, M. G. Knepley, L. C. McInnes, K. Rupp, B. F. Smith, S. Zampini, H. Zhang, H. Zhang, PETSc Users Manual, Tech. Rep. ANL-95/11 - Revision 3.7, Argonne National Laboratory, http://www.mcs.anl.gov/petsc, 2016.

[42] N. Aage, E. Andreassen, B. S. Lazarov, Topology optimization using PETSc: An easy-to-use, fully parallel, open source topology optimization framework, Structural and Multidisciplinary Optimization 51 (3) (2015) 565-572, ISSN 16151488, 1615147x.

[43] N. P. van Dijk, Formulation and implementation of stress-driven and/or strain-driven computational homogenization for finite strain, International Journal for Numerical Methods in Engineering 107 (12) (2016) 1009-1028.

[44] O. C. Zienkiewicz, R. L. Taylor, The finite element method for solid and structural mechanics, Elsevier Butterworth-Heinemann, Amsterdam; Boston, 2005.

[45] M. P. Bendsøe, O. Sigmund, Material interpolation schemes in topology optimization, Archive of Applied Mechanics 69 (9-10) (1999) $635-654$.

[46] B. S. Lazarov, O. Sigmund, Filters in topology optimization based on Helmholtz-type differential equations, International Journal for Numerical Methods in Engineering 86 (6) (2011) 765-781, ISSN 10970207, 00295981.

[47] A. Klarbring, N. Strömberg, Topology optimization of hyperelastic bodies including non-zero prescribed displacements, Structural and Multidisciplinary Optimization 47 (1) (2013) 37-48.

[48] K. Svanberg, The method of moving asymptotes - a new method for structural optimization, International Journal for Numerical Methods in Engineering 24 (2) (1987) 359-373.

[50] URL https://formlabs.com/3d-printers/form-2/

[51] URL https://formlabs.com/3d-printers/tech-specs/\#material-properties 